\title{
Hashtag-Forget: Using Social Media Ephemerality to Support Evolving Identities
}

\author{
Michal Luria \\ Human-Computer Interaction Institute, \\ Carnegie Mellon University \\ Instagram Research, Facebook \\ mluria@cs.cmu.edu
}

\author{
Nate Foulds \\ Instagram Research, Facebook \\ natefoulds@fb.com
}

\begin{abstract}
This work looks into the current state of sharing ephemeral versus permanent content on common social media platforms. Previous research has indicated that ephemerality of content, content that has an expiration date or time, can support users' identity construction. However, not much is known about whether current ephemeral interactions on social media are successful in doing so, or whether there are ephemerality-related design opportunities for social media platforms that can improve identity expression. In an 8-day qualitative diary study, participants reported when they posted on social media, and responded to questions about the type of content they shared, their motivation, and the content's ideal duration. We discuss our findings about short-term and long-term ephemerality as part of the social media experience, and the potential impact on the evolving identities of teenagers and young adults.
\end{abstract}

ACM Reference Format:

Michal Luria and Nate Foulds. 2021. Hashtag-Forget: Using Social Media Ephemerality to Support Evolving Identities. In CHI Conference on Human Factors in Computing Systems Extended Abstracts (CHI '21 Extended Abstracts), May 8-13, 2021, Yokohama, Japan. ACM, New York, NY, USA, 5 pages. https://doi.org/10.1145/3411763.3451734

\section{INTRODUCTION}

Permanently documented content has been the default across social media platforms since their rise. Previous work has shown that content permanence has many advantages, such as sustaining knowledge over time, supporting communication, and growing content [14]. In recent years, however, social media have also introduced ephemerality of digital content, where people can share content that has an expiration date and eventually disappears. This alternative was followed by research, which resulted in a better understanding of the value of forgetting and being forgotten [4].

For example, previous work has shown that ephemerality has the potential to enable users to share more spontaneously, and to provide a space for individuals to explore and evolve their identity $[4,6,10]$. However, it also showed that current social media does not fully realize this potential [4]. Not much is known about how, exactly, social media platforms should best leverage ephemerality

\section{(c) (i) $\ominus$}

This work is licensed under a Creative Commons Attribution-NoDerivs Internationa 4.0 License.

CHI '21 Extended Abstracts, May 8-13, 2021, Yokohama, Japan

(c) 2021 Copyright held by the owner/author(s).

ACM ISBN 978-1-4503-8095-9/21/05.

https://doi.org/10.1145/3411763.3451734 and their current platforms to fully support for identity exploration. The goal of this research effort is to provide initial indication on opportunities within this design space.

We conducted an 8-day diary study with teenagers and young adults to better understand the current role of ephemerality in content sharing on social media, and to identify new ephemeralityrelated design opportunities. Participants in the study were asked to share occurrences in which they posted on social media, or events in which they considered doing so, and for any type of postboth ephemeral and non-ephemeral ones. We were particularly interested in learning about why participants desired ephemerality for some content (commonly using "Stories" features on social media platforms-a feature that allows to share content only for the duration of 24 hours), and its potential impact on their selfperception and presentation.

Our findings surface two opportunities for ephemerality changes in design: Short-term and long-term ephemerality. Our main finding is regarding short-term ephemerality. We find that the current convention of 24 hours for ephemeral content does not necessarily provide a care-free space for young individuals to explore selfpresentations-participants perceived that 24 hours is long enough for almost all their followers to see. In contrast, our findings suggest evidence that shorter ephemerality, of 6-12 hours, may limit exposure and better support identity exploration. For example, participants may attempt to share content that is different to what they usually share, revealing and "trying out" new parts of their evolving identity.

Long-term ephemerality suggests to add an "expiration date" to content that is currently designed as permanent, for example, content on social media "Feeds." This feature exists on the WeChat platform that is widespread in South-East Asia. We examine the potential to extend this design choice to young audiences primarily in the United States, and on other social media platforms. We find that teenagers and young adults experience identity change over their time on social media, which creates a growing need to delete or archive previous, permanent content. Automatic archiving could mitigate concerns and cognitive effort of thinking about what is publicly shared about oneself at every given moment.

We conclude with recommendations for both short-term and long-term ephemerality for current social media platforms with large adoption by teenagers and young adults. We discuss how platforms can provide young audiences with the ability to forget and be forgotten, and thus give their digital identities space to change and evolve. Finally, we discuss some of the limitations and potential tradeoffs of doing so. 


\section{LITERATURE REVIEW}

Ephemerality, based on the Greek word ephemeros (lasting but one day), has been a key aspect of the widely used Snapchat and Instagram Stories features since their launch. A recent data analysis showed that ephemeral content, content that eventually disappears, was the number one contributor to continued use of Stories on Instagram [8].

In the literature we find two main categories that define ephemerality of digital content: "short-term ephemerality," a term we use to refer to content that can be viewed for up to 24 hours before it expires, and "long-term ephemerality," a term we use to refer to content that is semi-permanent, and that can last for several months before it is no longer publicly available.

\subsection{Short-Term Ephemerality}

Ephemerality keeps the volume of content on social media from getting overwhelming, and can reduce both mental load and physical load on the device's memory [13]. It gives a sense of timelinessStories are where users expect to see the most recent information about the people and things they care about. The sense of timeliness likely comes from both Stories' ephemerality and the type of content that is being shared [15].

Previous research pointed out that on the one hand, ephemerality on social media encourages users to share a broader, deeper and more diverse range of topics on social media due to its temporality [12]. On the other hand, it is not without concern of losing valuable content. In interviews about Stories use, some participants indicated that they frequently forget to save content on Snapchat, for example, and later regret it. Some even mentioned switching to other social media platforms to avoid potential loss of social information [12].

In recent years however, it seems that sharing on social media platforms has become a more careful and self-aware practice $[7,10]$. Several reasons, described in a recent study about the social media platform "WeChat," may have contributed to this change [7]: (1) People's networks have expanded and diversified beyond their close circles, making them more aware of what they are posting; (2) People mature over the years and tend to perceive their previous sharing habits as "silly" and "childish"; (3) People feel that too much information is no longer appreciated on social media and is bothersome, and therefore they have learned to "talk less. [7]"

Goffman theorized that self-presentation is like a "front stage," where people can present themselves based on their environment's norms, context and expectations. To continue this metaphor, Goffman has also describes a "backstage." This is where people might "rehearse," and more freely explore their identity, without having to worry about their self-presentation and judgement of others [6]. It seems that ephemeral social media was able to initially provide a "backstage" for users, but recent research suggests that current interactions on social media do not quite address the need for this kind of exploratory environment. Rather, research revealed that while Stories' ephemerality allows some opportunity to reconsider one's self-presentation, it is neither truly "backstage" nor a polished "front stage." Instead, it is described through the metaphor of an improvisational performance [10]-is is not as structured and "perfect" as a front stage, but also far from being "backstage."
From this we can conclude that for young people, the need to "rehearse" a range of content types, and figure out the current identity they desire when they present themselves on digital media is only addressed to a limited capacity [10]. We focus on this need as a goal for designing ephemeral interactions.

\subsection{Long-term Ephemerality}

The term "long-term ephemerality" addresses the opportunity to make some content ephemeral over a longer period of time (for example, to have some content disappear after a few weeks or months online). This is an approach that intends to counter data longevity and the feeling that anything that is posted online is going to last forever. Previous work has supported the relationship between ephemerality and privacy, showing that data longevity raises a sense of uncertainty and risk [1], especially privacy-related risk [11]. Some research suggested that participants had a stronger sense of privacy when content was automatically removed from public view by default [15]

Research by Bayer and colleagues also showed that Snapchat ephemeral messages were successful in addressing people's need to "go off record." Messaging on Snapchat was perceived as somewhat similar to face-to-face interactions in that you could only experience them once, in real time [2].

Finally, scholars have previously argued that automatic data persistence can lead to conflict within the perception of one's own identity. While individuals are likely to change their self-perception over time, data longevity tends to fixate the narrative about past selves in a particular way, and gives their community the ability to look back into one's history, also fixating on the same narrative [9].

A recent case-study examined the "Time Limit" feature that is available for WeChat Moments users [7]. This setting provides a form of "long-term ephemerality" on users' profiles, where users can decide how far back others can see on their profiles: 3 days, 1 month, 6 months or forever. The study showed that this feature was used by WeChat users to resolve inconsistencies between their old and current self-presentation; it allowed them to feel more control over their content and desired self-presentation by removing older content from public view, but still keeping a private record for themselves. This design strikes the balance between supporting users' need to express their identity and their need to maintain an archive of previous experiences [7]. In our work we examine how long-term ephemerality on popular social media platforms might play out and be perceived by participants.

\section{METHOD}

In order to examine the relationship between ephemerality and content and the impact of ephemerality on identity, we used the qualitative and exploratory method of a diary study. In diary studies, participants are given either a digital or a physical diary with instructions to make "entries" about a particular topic. It allows people to reflect on specific behaviors in-context, and share with researchers when a relevant moment appears, as opposed to more recall-based data collection formats. We conducted the study through an online platform designed specifically for digital diary studies (dscout), and enables researchers to recruit, run the procedure and analyze the data on a single platform [5]. 
In our study, for a duration of 8 days, participants were asked to make a "diary entry" in a digital diary when they either posted something on social media, or considered posting but decide not to. Participants were asked to upload the content that they shared on social media as part of their digital diary entry, and to reflect on some aspects of their behavior, such as why they posted on the platform they posted to, what responses they hope to receive, and what would be the ideal duration for this content if they could change it. Participants were required to make at least three entries in total throughout their 8-day study participation.

In addition to the diary portion of the study, we followed up with semi-structured interviews with 7 participants to clarify, deepen, and better understand some of the findings. For example, we asked participants what kind of content is included in most of their "Story" posts and why they think that is, or we asked them what content that is shared by others they enjoy most. The interviews were conducted remotely through video calls.

\subsection{Participants}

We recruited 45 participants for this study from the dscout platform participant pool. Participants were asked to answer a short screener to ensure they fall within the targeted population for this study. We screened for participants who were active on social media (posted at least once every two weeks), and participants who use Instagram regularly, as this was the platform we focused on for this diary study. We also aimed to recruit participants who use the "Stories" feature on other popular social media platforms, to better understand when ephemerality is used and for what type of content. We selected the final group of participants to be diverse based on self-reported factors such as location, age, gender, social media usage, ethnicity, and socioeconomic status. Participants were monetarily compensated for their participation.

Seventeen participants were teenagers between the ages of 14-17 $(\mathrm{m}=16.12)$, and 28 participants were young adults, ages $18-24(\mathrm{~m}$ $=20.32$ ). All COPA guidelines for participants under the age of 18 were followed, and parental consent was collected. Twenty-five participants self-identified as female, 19 participants self-identified as male, and one participant self-identified as non-binary. Slightly more than half of participants were based in the United States (24 participants), while the rest were sampled from across the world: Canada (6), Brazil (4), UK (3), Australia (2), Poland (1), Portugal (1), Germany (1), Spain (1), Mexico (1) and Guatemala (1).

\subsection{Procedure}

Once a diverse set of participants was selected, they were invited to begin our virtual diary study. Participants had to complete a total of three parts of the study: (1) An introductory questionnaire where they were asked about their habits on social media; (2) a week-long logging activity every time they posted on social media, or considered posting but decided not to (at least 3 entries were required); (c) a concluding entry about their experience, that included reflecting on the content they posted during the week of logging activity. All participants opted into participation after receiving detailed information about this procedure, and agreed to share some of the content they uploaded to social media as part of the study.
After being selected, participants were invited to begin the study online as soon as they were ready. In the first, introductory entry, they were asked about the frequency of their social media use, how they use the different platforms and features, and what kind of content they typically post.

The majority of data was submitted in the second part of the study. For this part, participants were asked to upload "diary entries" with content that they had uploaded to social media, and to share their reflection on it. First, they were asked to either take a snapshot of their post (for images), or take a video recording of their post (for videos). They were then asked to video record themselves ("selfievideo") and discuss why they posted the content they posted, and why they selected that specific medium. Finally, participants were asked to answer a few more brief questions about the post, such as, "what would be the ideal duration for this kind of content?", "what is the best response you expect for the content you posted?" and "what is the worst response that you expect for the content you posted?" All participants posted between 3-6 entries for part two of the study ( $\mathrm{M}=3.44$ entries).

In the last part, participants were asked to review and reflect on their social media posts throughout this week. Some questions included: "which post were you most satisfied with?", "during this week, did you delete any content after posting it?", and "did you save any of the content you posted onto your phone?".

This 3-part diary study resulted in a total of 156 posts on social media by participants, and a total of 247 diary entries. Over the course of 8 days, the primary researcher was in contact with participants through direct messages on the diary study platform. Participants could ask clarification questions at any time. Occasionally, the researcher sent participants reminders to ensure they spread out their entry uploads throughout the duration of the study.

\subsection{Follow-up Interviews}

After the diary study was completed, 7 participants were asked to participate in remote, one-on-one interviews with the researcher for additional compensation. Participants were selected based on a combination of diversity, availability, and the depth and clarity of reflections they shared during the diary study portion. During the interviews, participants were asked to further elaborate on some of their choices throughout the study, as well as additional questions about how they share and consume social media.

\subsection{Analysis}

The data for analysis consisted of verbal responses, written responses, and interview data (we did not conduct a content analysis screenshots and videos participants shared). All verbal responses were transcribed and analyzed together with written responses using a thematic coding approach [3] to identify themes-we used an inductive approach to crystallize a set of codes, continued with a combined inductive and deductive approach to find new codes, and lastly used a deductive approach for a final review of the data.

\section{FINDINGS}

We report our findings in two categories: Finding on short-term ephemerality on social media, and findings on long-term ephemerality on social media. 


\subsection{Short-Term Ephemerality}

$26.6 \%$ of participants expressed a desire to share the content they shared for the default 24 hours for a shorter duration of about 612 hours ( 12 participants from the total $n=45$, and for 19 posts in total.) The rest of participants felt comfortable with the current 24 hour time-frame for the content they uploaded, claiming it is just long enough for others to see. Participants were not interested in ephemerally that is shorter than 6 hours.

The fact that a quarter of participants desired an option of 6-12 hours in this short 8-day study period suggests an unanswered need for including ephemerality that is shorter than the standard 24 hour option, but longer than alternative social media content that last seconds or minutes.

When looking deeper into instances where a 6-12 hour duration was preferred, participants suggested three primary reasons: First, some participants said that posting for a shorter period of time would seem more in the moment and more exclusive than a 24 hour post [P36]. [P03] explained that she just wanted to share a small moment, an insignificant part of her day. Thus, the ideal time for it would be only a few hours. P30 mentioned that the video she shared was intended to make other people laugh, so it doesn't need a lot of time to be seen by as many people as possible. Most participants agreed that while they assume many of their friends will see their content, it is not important for them that everyone sees it.

Another common reasoning for wanting shorter ephemerality was using same-day logic-for example, when a user shared something in the morning, it made sense for them that the content would last until the end of the day. For example, [P03] shared a photo from brunch, and said that having it around the next day wouldn't make much sense.

Finally, participants desired shorter ephemerality when the content itself felt particularly personal. [P21] shared a funny selfie that he had to take due to a lost bet with his friends. The participant said that the content is very personal, which is why for this purpose, he would prefer to be able to limit the time for shorter than 24 hours.

The common aspect of all the content that participants desired to share for shorter periods of time was that it was personal content about them, about their life, or their activities (rather than content about others or about the news, which constituted $52 \%$ of all social media posts shared in this study.) The content that participants wanted to share for a shorter duration was always personal, and frequently silly or intimate. This suggests that shorter ephemerality with limited exposure (but not too limited), could be beneficial for "improvisational performance" [10] and exploration of new identities or intimate moments that individuals felt too self-aware to share for a longer, 24-hour, duration.

Another finding that aligns with this insight is that several participants who did not post content but considered doing so, said that the ideal duration would have been 6-12 hours long. Participants explained that the content did not seem important enough [P04] or that they worried their friends would judge them [P17]. [P08] recalled a time when she posted a Story that asked others for music recommendations, after convincing herself not the be shy about it. However, a short while after posting she still felt selfaware, and decided to delete it, although she felt there is some shame associated with doing so. Shorter ephemerality could allow users to express new interactions and under-explored aspects of their changing identity through social media, without having to delete content prior to its intended expiration time (which was often accompanied by discomfort and even shame).

\subsection{Long-Term Ephemerality}

Teenagers and young adults report experiencing change over time on social media, and a growing need to delete permanent content as their identity evolves. Our findings echo previous work that found value in long-term ephemerality on the WeChat Moments platform, and extends this value to users in the United States and in other parts of the world, and across other, more visual social media platforms such as Instagram and Snapchat.

[P08] described how her teenage self used to post a lot of content, almost everything that had happened to her. Going into high school and seeing others' behaviors made her come to the conclusion that "oversharing" is perceived negatively. She therefore deleted previous content, and changed her sharing behavior from then on. Similarly, P31 admitted to occasionally going on a "deletion spree" of old content, for example, from middle school. P40 detailed his own experience with old content deletion. After beginning high school, he looked back and felt embarrassed at the content he had on his profile. He decided nobody should be able to see this content anymore, and deleted it all. Participants were especially prone to "bulk deleting" content during life or location transitions, when they expected to meet many new people on a daily basis and have some shift in their perceived (and shared) identity (such as transitioning to a new school, from middle school to high school, or from high school to college.)

These findings support an opportunity for "long-term ephemerality" for all social media content. Evidence suggests that introducing long-term ephemerality, where individuals can set an expiration date to automatically archive all content (e.g., after 3 months), can better support the evolving identities of teenagers and young adults. Participants not only described having to go through old content to delete large amounts of posts, but also sometimes being shocked by what is "out there" and quickly deleting with a strong sense of embarrassment for not noticing earlier. Long-term ephemerality is promising to help reduce the anxiety and mental load that comes with having to always track and groom their public online identity.

That said, an alternative might be to provide people with reminders to review older content-more research is needed to identify which approach would better support young individuals and their evolving identities.

\section{DISCUSSION AND DESIGN OPPORTUNITIES}

In this work, we identified findings about how short-term and longterm ephemerality can be put to better use to support the evolving identities of teenagers and young adults.

Participants indicated that Story ephemerality is valuable to post content that they do not permanently want on their profiles. However, the current 24 hour ephemerality model on Instagram and Snapchat is perceived as quite long-long enough for all participants' followers, or at least most of their followers to be able to view. Some participants suggested that shorter ephemerality, 
that would ideally last between 6-12 hours, might be better for attempting new kinds of content sharing-personal content, "socially risky" content (such as something silly or private), or simply content that is perceived as "in-the-moment." Participants viewed limited ephemerality as a means to not only constrain the audience quantity due to the more limited time window, but also to constrain the audience proximity. This is because participants assumed that their closest fiends and family will view their content as soon as they see it is available, while more distant circles are more likely to delay viewing their content.

Design Recommendation: We suggest that an option to share on social media for a shorter duration (for 6-12 hours) might better support personal content and identity exploration, and that it would allow individuals to organically share with their closer social circles. Less than 6 hours was mostly perceived as "disappearing content," which usually serves a different purpose. Thus, 6-12 hour sharing is likely to work best for identity exploration on platforms that already have a similar time-frame for content (24 hours), such as social media Stories.

Second, participants indicated that much of their "permanent" content, content that they post on their Instagram Feed for example, has to be reviewed and "groomed" from time to time. Occasionally, participants go on "deletion sprees" and delete many photos at once. They also expressed a sense of embarrassment when they realized old content that no longer represents them is still available for others to view. We see an opportunity here to explore and enable long-term ephemerality for all permanent content on social media. By allowing participants to set an expiration date for all content, after which it is automatically archived, social media platforms can better support users' constantly evolving identities, especially teenagers and young adults. This could be particularly important in "life transition" moments.

Our work suggests initial evidence that long-term ephemerality can positively impact young people's experience on social media. Future research can shed light on the experience of using possible long-term ephemerality features on one's own content.

Design Recommendation: We believe that a long-term ephemerality feature for all social media content can help relieve some of the anxiety young people face due to maintaining awareness of what content is publicly shared at every given moment and on every platform, and what that might portray about their identity. This idea should be examined in the context of current permanent content on social media, primarily social media "feeds."

While our findings suggest that interactions of ephemerality are positive for social media content, they are not without drawbacks. First, prior literature highlights some of the positive aspects of data longevity, such as sustaining knowledge over time and supporting interpersonal communication [14]. Second, Cardell explains about ephemerality that "this kind of self-narration might seem as if it offers freedom from surveillance, publicness, and the archival nature of other social media forums. But how free can you feel when you are compelled to log on each day to quell the anxiety of losing your streak or missing out on content with a tight expiration date?" [4]. In other words, ephemerality also introduces stress around socially "missing out," and creates a sense of urgency. This could be exacerbated if some content lasts even less than 24 hours. Thus, more work is required to better understand which of the two has stronger effects, and to explore interaction design opportunities to mitigate the negative consequences of "the fear of missing out" of short-living social media content.

To summarize, ephemerality is an important component for social media content and for supporting young people's evolving identities, both through short-term exploration and through limiting long-term documentation. We hope that the findings presented here will encourage social media platforms to embrace data ephemerality, and to give their users more opportunities to change and evolve over time.

\section{REFERENCES}

[1] Alessandro Acquisti, Laura Brandimarte, and George Loewenstein. 2015. Privacy and human behavior in the age of information. Science 347, 6221 (2015), 509-514.

[2] Joseph B Bayer, Nicole B Ellison, Sarita Y Schoenebeck, and Emily B Falk. 2016. Sharing the small moments: ephemeral social interaction on Snapchat. Information, Communication \& Society 19, 7 (2016), 956-977.

[3] Virginia Braun and Victoria Clarke. 2012. Thematic analysis. (2012).

[4] Kylie Cardell, Kate Douglas, and Emma Maguire. 2017. Social media and ephemeral narratives as memoir. Mediating Memory: Tracing the Limits of Memoir (2017), 157-172.

[5] Dscout. accessed September 09, 2020. . https://dscout.com/

[6] Erving Goffman et al. 1978. The presentation of self in everyday life. Harmondsworth London.

[7] Xiaoyun Huang, Jessica Vitak, and Yla Tausczik. 2020. "You Don't Have To Know My Past": How WeChat Moments Users Manage Their Evolving Self-Presentation. In Proceedings of the 2020 CHI Conference on Human Factors in Computing Systems. $1-13$.

[8] İbrahim KIRCOVA, Fatih PINARBAŞI, and Şirin Gizem KÖSE. 2020. Understanding Ephemeral Social Media Through Instagram Stories: A Marketing Perspective. Business \& Management Studies: An International fournal 8, 2 (2020), 2173-2192.

[9] Viktor Mayer-Schönberger. 2011. Delete: The virtue of forgetting in the digital age. Princeton University Press.

[10] Sarah McRoberts, Haiwei Ma, Andrew Hall, and Svetlana Yarosh. 2017. Share first, save later: Performance of self through Snapchat stories. In Proceedings of the 2017 CHI Conference on Human Factors in Computing Systems. 6902-6911.

[11] George R Milne, George Pettinico, Fatima M Hajjat, and Ereni Markos. 2017. Information sensitivity typology: Mapping the degree and type of risk consumers perceive in personal data sharing. Fournal of Consumer Affairs 51, 1 (2017), 133-161.

[12] Tina Natalie Morlok, Ioanna Constantiou, and Thomas Hess. 2018. Gone for better or for worse? Exploring the dual nature of ephemerality on social media platforms. (2018).

[13] William Odom, Richard Banks, David Kirk, Richard Harper, Siân Lindley, and Abigail Sellen. 2012. Technology heirlooms? Considerations for passing down and inheriting digital materials. In Proceedings of the SIGCHI Conference on Human Factors in computing systems. 337-346.

[14] Jeffrey W Treem and Paul M Leonardi. 2013. Social media use in organizations: Exploring the affordances of visibility, editability, persistence, and association. Annals of the International Communication Association 36, 1 (2013), 143-189.

[15] Heng Xu, Hock-Hai Teo, Bernard CY Tan, and Ritu Agarwal. 2012. Research note-effects of individual self-protection, industry self-regulation, and government regulation on privacy concerns: a study of location-based services. Information Systems Research 23, 4 (2012), 1342-1363. 